\title{
Low toxic maghemite nanoparticles for theranostic applications
}

\author{
This article was published in the following Dove Press journal: \\ International Journal of Nanomedicine \\ 31 August 2017 \\ Number of times this article has been viewed
}

\section{Elena A Kuchma* \\ Peter V Zolotukhin \\ Anna A Belanova \\ Mikhail A Soldatov \\ Tatiana A Lastovina \\ Stanislav P Kubrin \\ Anatoliy $\vee$ Nikolsky \\ Lidia I Mirmikova \\ Alexander V Soldatov*}

The Smart Materials Research Center, Southern Federal University of Russia, Rostov-on-Don, Russia

*These authors contributed equally to this work
Correspondence: Alexander V Soldatov; Elena A Kuchma

The Smart Materials Research Center, Southern Federal University of Russia, Sladkova 178, Rostov-on-Don 344090, Russia

Email soldatov@sfedu.ru; elenaku4ma@ yandex.ru
Background: Iron oxide nanoparticles have numerous and versatile biological properties, ranging from direct and immediate biochemical effects to prolonged influences on tissues. Most applications have strict requirements with respect to the chemical and physical properties of such agents. Therefore, developing rational design methods of synthesis of iron oxide nanoparticles remains of vital importance in nanobiomedicine.

Methods: Low toxic superparamagnetic iron oxide nanoparticles (SPIONs) for theranostic applications in oncology having spherical shape and maghemite structure were produced using the fast microwave synthesis technique and were fully characterized by several complementary methods (transmission electron microscopy [TEM], X-ray diffraction [XRD], dynamic light scattering [DLS], X-ray photoelectron spectroscopy [XPS], X-ray absorption near edge structure [XANES], Mossbauer spectroscopy, and HeLa cells toxicity testing).

Results: TEM showed that the majority of the obtained nanoparticles were almost spherical and did not exceed $20 \mathrm{~nm}$ in diameter. The averaged DLS hydrodynamic size was found to be $\sim 33 \mathrm{~nm}$, while that of nanocrystallites estimated by XRD was $\sim 16 \mathrm{~nm}$. Both XRD and XPS studies evidenced the maghemite $\left(\gamma-\mathrm{Fe}_{2} \mathrm{O}_{3}\right)$ atomic and electronic structure of the synthesized nanoparticles. The XANES data analysis demonstrated the structure of the nanoparticles being similar to that of macroscopic maghemite. The Mossbauer spectroscopy revealed the $\gamma-\mathrm{Fe}_{2} \mathrm{O}_{3}$ phase of the nanoparticles and vibration magnetometry study showed that reactive oxygen species in HeLa cells are generated both in the cytoplasm and the nucleus.

Conclusion: Quasispherical Fe ${ }^{3+}$ SPIONs having the maghemite structure with the average size of $16 \mathrm{~nm}$ obtained by using the fast microwave synthesis technique are expected to be of great value for theranostic applications in oncology and multimodal anticancer therapy.

Keywords: magnetic nanoparticles, SPIONs, maghemite, theranostics in oncology, toxicity, ROS

\section{Introduction}

Magnetic iron oxide nanoparticles are very promising candidates for the applications in future nanomedicine, ${ }^{1,2}$ in terms of developing smart therapy approaches. ${ }^{3}$ Superparamagnetic iron oxide nanoparticles (SPIONs) could be used in a combined manner, ie, not only as contrast agents for diagnostics and treatment monitoring but also as active substances for medical treatment. ${ }^{4}$ In many cases, SPIONs represent a novel platform for simultaneous therapy and diagnostics (theranostics) in oncology. ${ }^{5-7}$ Recently, it has been found that iron oxide nanoparticles are even capable of inhibiting tumor growth by stimulating macrophages. ${ }^{8}$

The properties of SPIONs are known to be size dependent, and consequently, the effect that they produce on tissues is varied..$^{9,10}$ Another important parameter responsible for toxicity of the SPIONs is the shape of these nanoparticles. It was 
shown that rod-shaped nanoparticles were more toxic than the sphere-shaped ones. ${ }^{11}$ One more tuning parameter could be the type of iron oxide structure, for instance, one could grow the SPIONs of $\mathrm{Fe}_{3} \mathrm{O}_{4}$ or $\mathrm{Fe}_{2} \mathrm{O}_{3}$ modification. ${ }^{12}$ It was found that the oxidation state of iron $\left(\mathrm{Fe}^{2+}\right.$ or $\left.\mathrm{Fe}^{3+}\right)$ in SPIONs may affect the cytotoxic properties of the nanoparticles in biological tissues. ${ }^{13}$ Sometimes, maghemite $\left(\mathrm{Fe}_{2} \mathrm{O}_{3}\right)$ nanoparticles show more significant genotoxicity than those of magnetite $\left(\mathrm{Fe}_{3} \mathrm{O}_{4}\right) \cdot{ }^{14}$ However, in other cases, $\mathrm{Fe}_{2} \mathrm{O}_{3}$ SPIONs do not induce any significant DNA damage. ${ }^{15}$ In the present work, we studied the characteristics (including cytotoxicity) of spherically shaped $\mathrm{Fe}_{2} \mathrm{O}_{3}$ SPIONs synthesized by the fast microwave-assisted hydrothermal method.

\section{Methods}

\section{Ethics}

All experiments were in accordance with local regulations and approved by the authorized joint scientific and ethical committee of the Southern Federal University, Academy of Biology and Biotechnology, and "Smart Materials" International Research Centre.

\section{Synthesis of SPIONs}

Magnetic nanoparticles were synthesized by means of the microwave-assisted coprecipitation using the modified scheme of Blanco-Andujar et al. ${ }^{16}$ The synthesis was carried out as follows. A solution of $1,112 \mathrm{~g}$ of $\mathrm{FeSO}_{4} \cdot 6 \mathrm{H}_{2} \mathrm{O}$ (II) and $21,624 \mathrm{~g}$ of $\mathrm{FeCl}_{3} \cdot 6 \mathrm{H}_{2} \mathrm{O}$ with $40 \mathrm{~mL}$ of water was obtained. The solution was blown with argon for $40 \mathrm{~min}$, in order to remove oxygen from the reaction medium. Next, a flask with the solution was placed in a microwave (Discover SP; CEM Corporation, Matthews, NC, USA). After the temperature reached $60^{\circ} \mathrm{C}$, the solution containing 33,921 $\mathrm{g}$ of $\mathrm{Na}_{2} \mathrm{CO}_{3}$ dissolved in $10 \mathrm{~mL}$ of water (also earlier blown with argon) was added to the reaction medium for $2.5 \mathrm{~min}$. With continuous stirring, the reaction medium was maintained at $60^{\circ} \mathrm{C}$ for an hour. Then, 1,536 $\mathrm{g}$ of citric acid dissolved in $5 \mathrm{~mL}$ of water was added for $2.5 \mathrm{~min}$ and kept in the microwave for another $60 \mathrm{~min}$. The precipitate was separated by centrifugation at 22,000 rpm for $40 \mathrm{~min}$, which was then followed by multiple washing by means of bidistilled water and drying at $40^{\circ} \mathrm{C}$ in a vacuum chamber overnight. ${ }^{16}$

\section{Characterization}

The X-ray diffraction (XRD) of the obtained sample was measured by the 2D PHASER (Bruker AXS Inc., Madison, WI, USA) using $\mathrm{Cu} \mathrm{K} \alpha$ radiation $(\lambda=1,540,562 \AA)$ at $30 \mathrm{kV}$ and $10 \mathrm{~mA}$. The sizes of the obtained nanocrystallites were calculated by the Scherrer equation. The transmission electron microscope (TEM) Tecnai G2 Spirit BioTWIN (FEI, Hillsboro, OR, USA) with accelerating voltage of $120 \mathrm{kV}$ was exploited to carry out the electron microscopic studies. The hydrodynamic size of the synthesized particles was measured using the DLS technique, by the particle size analyzer NANO-flex (Microtrac, Krefeld, Germany).

The X-ray absorption near edge structure (XANES) spectra were measured at the ID26 beamline of the European Synchrotron Radiation Facility (ESRF) (Grenoble, France). To obtain high energy resolution XANES, a special setup was used (which includes additional X-ray spectrometer). ${ }^{17}$ Such a technique made it possible to get XANES spectra without core-hole broadening or smearing of the fine structures. ${ }^{18}$

The Mossbauer spectra were measured by using the MS1104Em Mossbauer spectrometer (Rostov-on-Don, Russia). The ${ }^{57} \mathrm{Co}$ in $\mathrm{Cr}$ matrix was used as a $\gamma$-ray source. The samples were cooled in the camera of helium cryostat CCS-850 (Janis Research, Inc., Woburn, MA, USA). The experimental spectra were fitted using the Spectr Relax software. ${ }^{19}$ The isomer shifts were calculated with respect to the metallic $\alpha$-Fe. The magnetic properties of the obtained material were examined by using the vibrating magnetometer VSM 7404 (LakeShore, USA).

The X-ray photoelectron spectra of synthesized magnetic nanoparticles were taken at room temperature using X-ray photoelectron microprobe ESCALAB 250. The spectra were excited with a monochromatized Al Ka-radiation ( $h v=1486.6 \mathrm{eV}$ ). A thin layer of finely powdered sample was deposed on a conductive carbon tape and fixed in a standard stainless steel sample holder. The XPS spectra were processed be means of Avantage software.

\section{Toxicity and cell viability testing}

For in vitro cytotoxicity testing, we used HeLa cell line as the model for the cytotoxicity test. The cells were subcultured in T25 flasks and further cultured for the analysis in 96-well plates (SPL Life Sciences, Pocheon-si, South Korea) in the GlutaMax DMEM (Thermo Fisher Scientific, Waltham, MA, USA) supplemented with $10 \%$ of fetal bovine serum (GE Healthcare UK Ltd, Little Chalfont, UK) and $0.05 \mu \mathrm{g} / \mathrm{mL}$ of gentamicin (Biokhimik JSC, Saransk, Russia). The cells were kept at $37^{\circ} \mathrm{C}$ and $5 \% \mathrm{CO}_{2}$, with passive humidification in the Sanyo MCO-18AC incubator (Panasonic, Moriguchi, Japan).

For the cell viability assay, the nanoparticle formulation was re-suspended in physiological saline, so as to reach the maximum possible concentration without forming a precipitate. The concentration obtained was $3 \mathrm{mg} / \mathrm{mL}$. Then, $5 \mu \mathrm{L}$ of the sample was added to $50 \mu \mathrm{L}$ of the culture medium into 
each well of the 96-well plate providing the final concentrations of the nanoparticles up to $0.3 \mathrm{mg} / \mathrm{mL}$.

The cell viability was assessed using the trypan blue exclusion assay during two different days: three biological replicates per day and two technical replicates per biological replicate for each nanoparticle sample. Briefly, the cells treated with the nanoparticles and control saline were detached with $0.25 \%$ trypsin-sodium ethylenediaminetetraacetate solution and mixed with an equal volume of $0.4 \%$ trypan blue (Thermo Fisher Scientific) and, after $2 \mathrm{~min}$ incubation, counted in the Goryaev chamber (the chamber is analogous to the hemocytometer; MiniMed LLC, Suponevo, Russia) under the Primo Star microscope (Carl Zeiss Meditec AG, Jena, Germany). Not $<300$ cells were counted in each technical replicate. The statistical calculations were performed using the SPSS 22 package. On the graph, the cell viability data are presented as mean $\pm \mathrm{SD}$. The data were analyzed for normality (the zero hypothesis was retained in all groups) using the Kolmogorov-Smirnov test and for statistical differences using nonparametric (Mann-Whitney criterion) and parametric analysis of variance test for crossconsistency.

To test how the nanoparticles affected cellular reactive oxygen species (ROS) productions, we used the CellROX Green and Orange reagents (Thermo Fisher Scientific). Briefly, the cells were grown on poly-lysine-covered coverslips in 24 -well plates. When the cells were $40 \%-50 \%$ confluent, the sample and vehicle were added to the respective wells ( $30 \mu \mathrm{L}$ of the sample to $300 \mu \mathrm{L}$ of the culture medium), and then, the cells were incubated for $24 \mathrm{~h}$ and stained according to the manufacturer's protocol. The cells were then imaged using the BX61 microscope (Olympus Corporation, Tokyo, Japan). It should be noted that the CellROX Green reagent

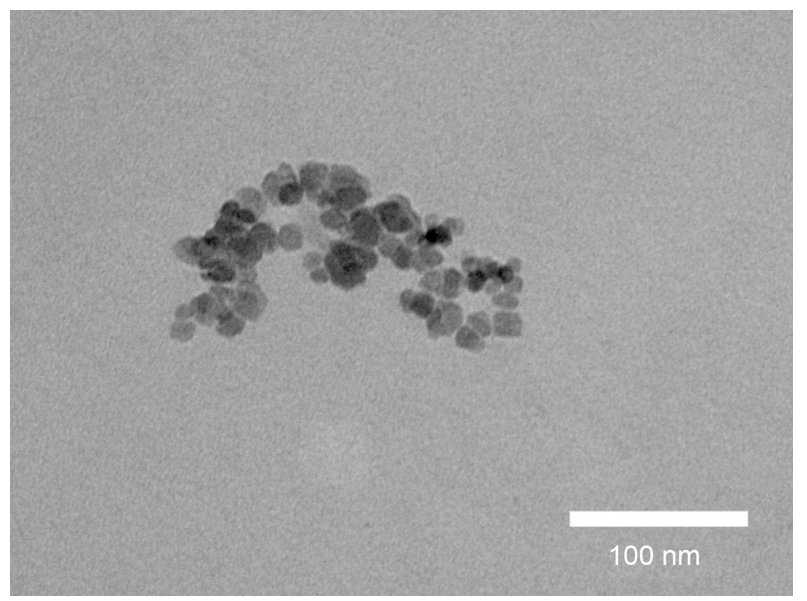

Figure I TEM images of the SPIONs synthesized using microwave-assisted coprecipitation.

Abbreviations: SPIONs, superparamagnetic iron oxide nanoparticles; TEM, transmission electron microscopy.

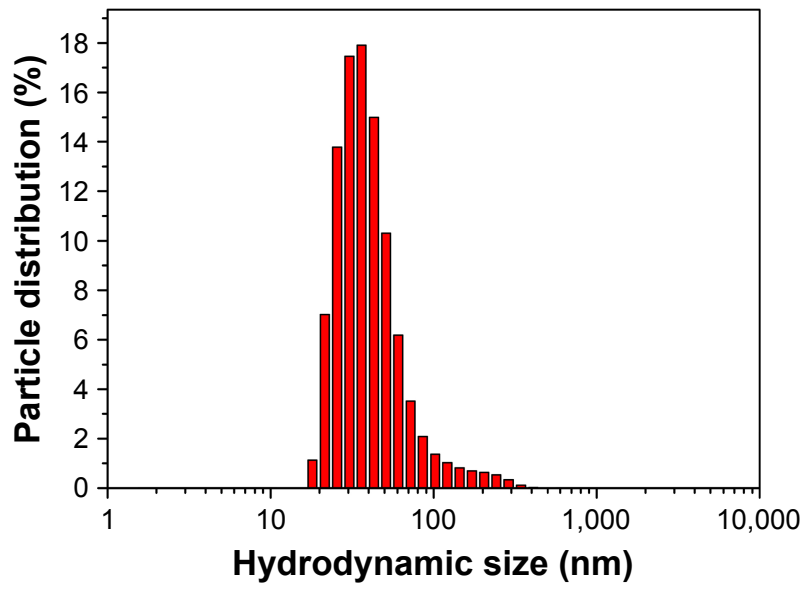

Figure 2 The size distribution of SPIONs revealed by DLS.

Note: The interval shown in red color indicates the hydrodynamic sizes of the nanoparticles.

Abbreviations: DLS, dynamic light scattering; SPIONs, superparamagnetic iron oxide nanoparticles.

reacts with nuclear and mitochondrial ROS, while CellROX Orange fluorescence is only activated by cytosolic ROS.

It should be stressed that HeLa cells were kindly provided by Southern Scientific Center of the Russian Academy of Sciences and validated by the cytogenetic (G-staining) and molecular genetic analyses.

\section{Results' characterization of nanoparticles}

The majority of the nanoparticles synthesized in the present work are almost spherical and do not exceed $20 \mathrm{~nm}$ (average size is $16 \mathrm{~nm})$. This can be seen in the pictures taken by means of TEM (Figure 1).

Using DLS, the hydrodynamic sizes of the obtained nanoparticles were measured and the average hydrodynamic size was found to be $33.6 \mathrm{~nm}$ (Figure 2).

The result of the XRD study is presented in Figure 3. The maximum peaks $2 \theta$ indicate that the spinel-type iron oxide structure is formed. Average-sized crystallites were

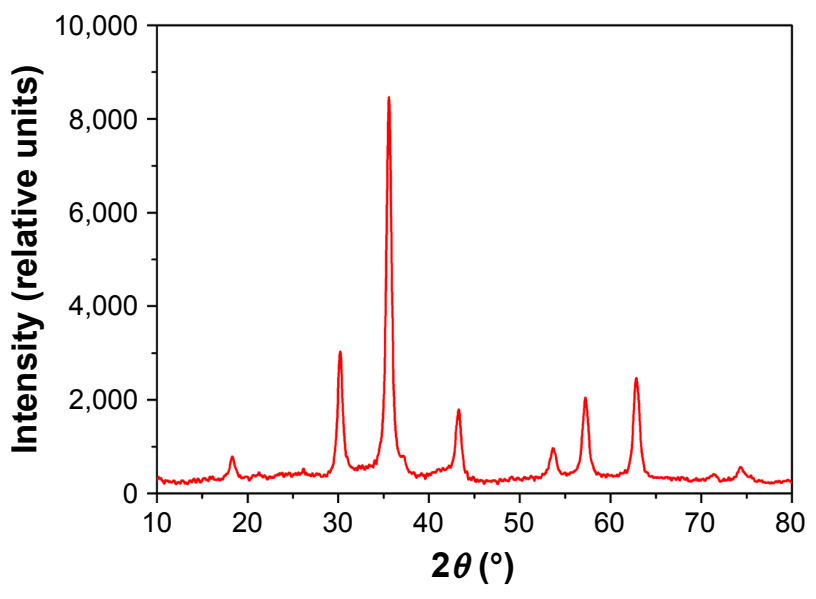

Figure 3 X-ray diffractogram of the synthesized iron oxide nanoparticles. 


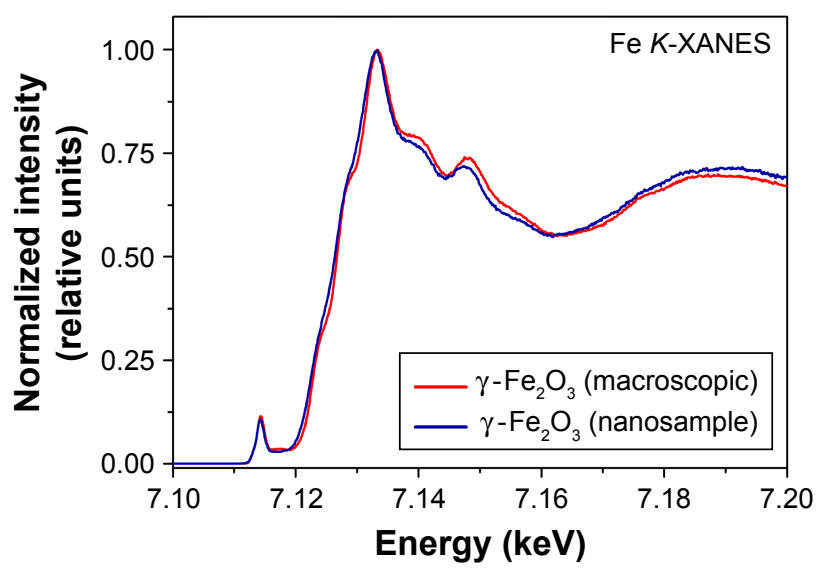

Figure 4 XANES method illustrates that the spectrum of the obtained nanoparticles coincides with the spectrum of the gamma phase $\mathrm{Fe}_{2} \mathrm{O}_{3}$ nanoparticles.

Abbreviation: XANES, X-ray absorption near edge structure.

estimated by means of the Debye-Scherrer equation. The $\mathrm{XRD}$ analysis shows that the synthesized nanoparticles have an averaged size of $16.2 \mathrm{~nm}$.

The XANES technique was applied to determine the electronic and crystal structure of the obtained nanomaterials. The analysis of the Fe K-edge XANES spectra (Figure 4) reveals that the synthesized SPIONs have a structure similar to that of the macroscopic gamma phase of $\mathrm{Fe}_{2} \mathrm{O}_{3}$ and the oxidation state of iron ions is $\mathrm{Fe}^{3+}$. The spectra obtained for the nanoparticles under study are rather similar to the previously measured spectra of the nanoparticles synthesized without microwave assistance..$^{20,21}$

XPS Fe2p spectrum of our sample is shown in Figure 5. As one can see, the Fe2p spectrum consists of two main peaks $\mathrm{A}$ and $\mathrm{C}$, corresponding to $2 \mathrm{p}_{3 / 2}$ and $2 \mathrm{p}_{1 / 2}$ states of Fe, arising from the spin-doublet splitting of $2 p$ level. The elemental composition performed by the analysis of XPS spectrum

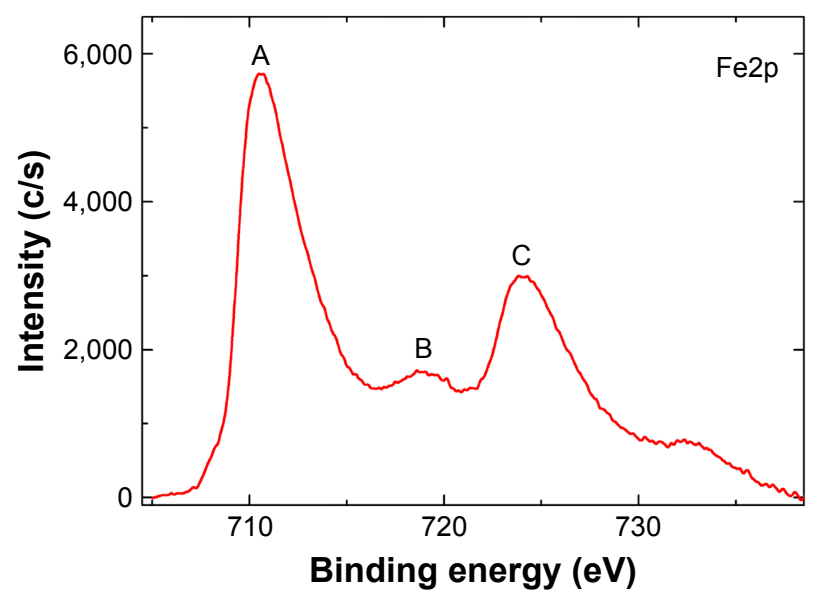

Figure 5 Experimental Fe2p XPS of the obtained SPIONs.

Abbreviations: c/s, counts per second; SPIONs, superparamagnetic iron oxide nanoparticles; XPS, X-ray photoelectron spectroscopy.
Table I Elemental composition of the SPIONs samples obtained by XPS

\begin{tabular}{lll}
\hline Elemental composition, atomic\% \\
\hline $\mathrm{Fe}$ & $\mathrm{O}$ & $\mathrm{C}$ \\
\hline $35.7 \pm 2.0$ & $50.2 \pm 2.7$ & $14.1 \pm 1.5$ \\
\hline
\end{tabular}

Abbreviations: SPIONs, superparamagnetic iron oxide nanoparticles; XPS, X-ray photoelectron spectroscopy.

is shown in Table 1. In our case, the energy position of the $\mathrm{Fe} 2 \mathrm{p}_{3 / 2}$ peak falls at $\sim 710.6 \mathrm{eV}$ that is rather close to the position of the $\mathrm{Fe} 2 \mathrm{p}_{3 / 2}$ peak in iron(III) compounds. ${ }^{22}$ However, the energy position of the $\mathrm{Fe} 2 \mathrm{p}_{3 / 2}$ peak cannot be the only criteria for determining the valence state of the iron ions in the powdered samples, prepared under air atmosphere.

One can look for the presence or absence of the characteristic feature in the spectrum of iron. This feature is a component $\mathrm{B}$, which is present in the Fe2p spectrum of our sample (Figure 5). This feature is located $8.3 \mathrm{eV}$ higher than the $\mathrm{Fe} 2 \mathrm{p}_{3 / 2}$ peak, and its presence is characteristic for the Fe2p XPS of $\mathrm{Fe}^{3+}$ compounds. ${ }^{23}$ Thus, the proximity of the $\mathrm{Fe} 2 \mathrm{p}_{3 / 2}$ energy position to the table value for $\mathrm{Fe}^{3+}$ compounds (compare to the example in Kozakov et $\mathrm{a}^{22}$ ) and especially the presence of the significant $\mathrm{B}$ peak can attribute the sample to maghemite $\left(\gamma-\mathrm{Fe}_{2} \mathrm{O}_{3}\right)$.

Figure 6 shows the Mossbauer spectra of SPIONs measured at both $14 \mathrm{~K}$ and room temperature. The Zeeman splitting is observed in both spectra, which indicates the magnetic state ordering. The line shape of the room temperature spectrum points to the presence of superparamagnetic relaxation. ${ }^{24}$ The isomer shift value $(\delta)$ of the sextet $\mathrm{S} 1$ corresponds to $\mathrm{Fe}^{3+}$ ions in the oxygen octahedron. ${ }^{25}$ The presence of the $\mathrm{Fe}^{3+}$ octahedron and tetrahedron sites indicates the $\gamma-\mathrm{Fe}_{2} \mathrm{O}_{3}$ phase.

Using a vibration magnetometer, we measured magnetic hysteresis loops of the synthesized nanoparticles. The powder samples were measured by the applied magnetic field, up to 20 Oe, at temperature $295 \mathrm{~K}$. The graph in Figure 7 shows the hysteresis loop, which characterizes the dependence of magnetic moment on the applied magnetic field. The maximum value of the magnetic moment of the synthesized nanoparticles was found to be $\sim 67.8 \mathrm{emu} / \mathrm{g}$. Judging by the hysteresis loop, we can conclude that the obtained nanoparticles appear to be superparamagnetic.

As seen in Figure 8, nanoparticles did not have any significant effects on the viability of the HeLa cells.

\section{Toxicity tests}

Previously, we had tested thoroughly $\mathrm{Fe}_{3} \mathrm{O}_{4}$ nanoparticles without colloid stabilizers resuspended in saline 

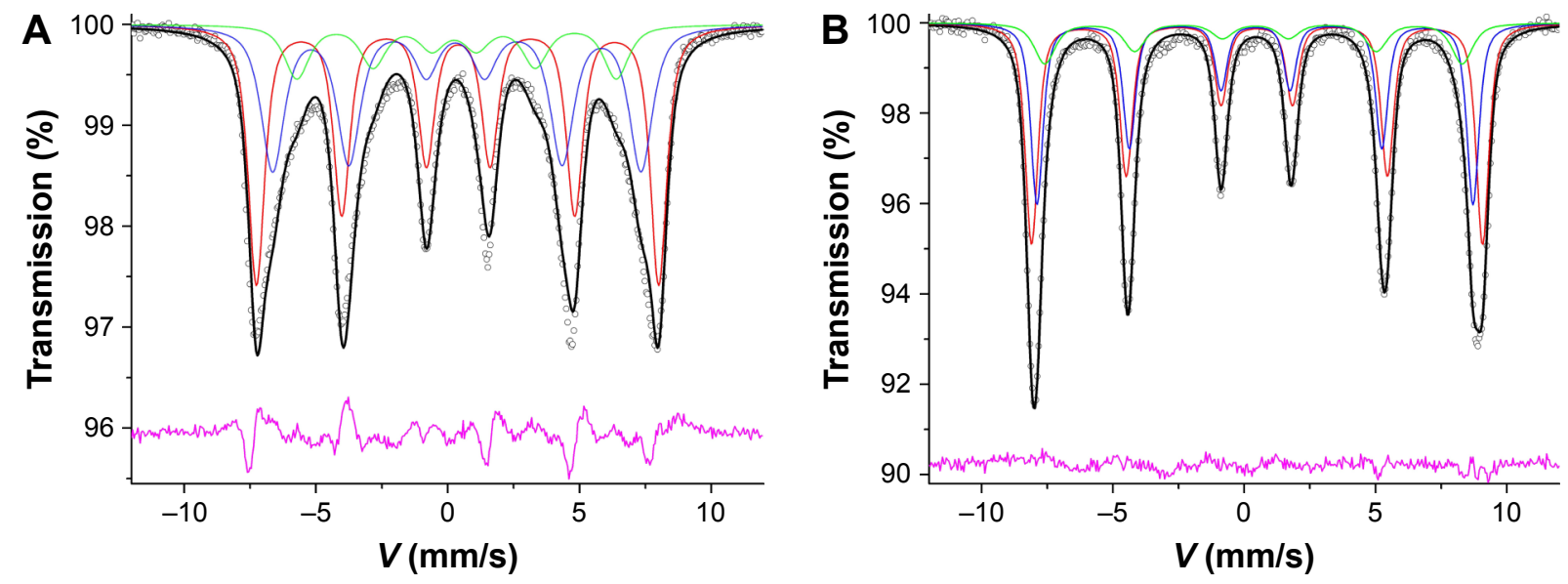

Figure 6 Mossbauer spectra of SPIONs taken at room temperature $(\mathbf{A})$ and temperature $14 \mathrm{~K}(\mathbf{B})$.

Note: The points mark the experimental data; the solid line is the result of fitting the spectrum by three sextets (green, blue, and red lines).

Abbreviation: SPIONs, superparamagnetic iron oxide nanoparticles.

(nonmodified; 50-100 nm in diameter; Sigma-Aldrich Co., St Louis, MO, USA). It was found that these nanoparticles did not exhibit any significant cytotoxicity, yet demonstrated substantial ROS promoting effect (data not shown). In the present study, we also performed the CellROX Green and Orange staining of the cells treated with the sample of the nanoparticles, because ROS formation induction is another option that can be used to reinforce thermotherapy and SPION-based drug delivery. ${ }^{26}$

As seen in Figure 9, the nanoparticles sample caused a profound increase in ROS generation in the HeLa cells - in both the cytoplasm and nucleus.

From our previous studies, it was evident that nonmodified $\mathrm{Fe}_{3} \mathrm{O}_{4}$ nanoparticles did not generally demonstrate induction of ROS in the nuclei of tumor cells. However, in this study, the $\gamma-\mathrm{Fe}_{2} \mathrm{O}_{3}$ nanoparticles greatly increased nuclear

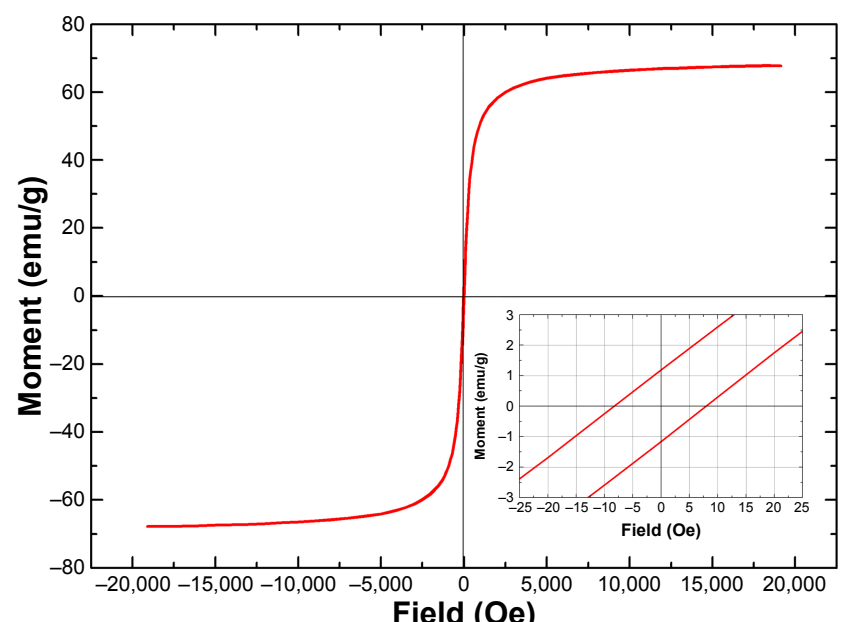

Figure 7 Hysteresis loop which characterizes the dependence of magnetic moment on the applied magnetic field.

Note: The insert shows enlarged region around zero magnetic field. fluorescence of the CellROX Green sensor. Moreover, as can be seen in Figure 9, the feature of the nuclear CellROX Green signal distribution pattern is granulation.

\section{Discussion}

As iron oxide nanoparticles are considered to be promising targeted delivery and release agents and theranostic agents, their own cytotoxicity is unwanted, unless the cytotoxicity mechanisms and character are worth risking. The reason is that the main benefits of using iron oxide nanoparticles are only true when their enhanced permeability and retention (EPR) effect is coupled with their capability to be locally activated using ultra-sound, magnetic impulses or heat. The EPR effect is never absolute: the particles will always be distributed over the body to some extent (not many delivery agents can reach $>5 \%$ of the injected dose per tumor, and the rest $95 \%$ distribute in normal tissues). ${ }^{27}$ Therefore, the lower the inherent cytotoxicity

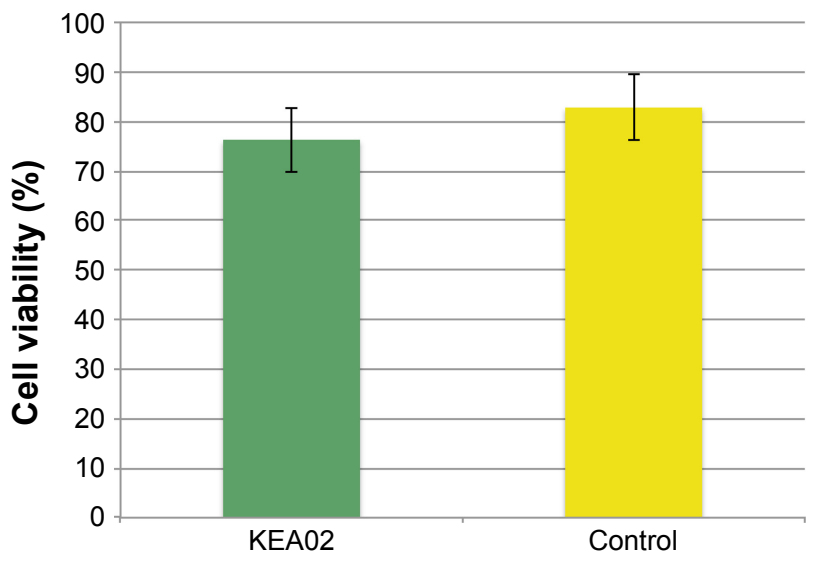

Figure 8 Cell viability after $24 \mathrm{~h}$ exposure to the synthesized iron oxide nanoparticles or saline (control). 

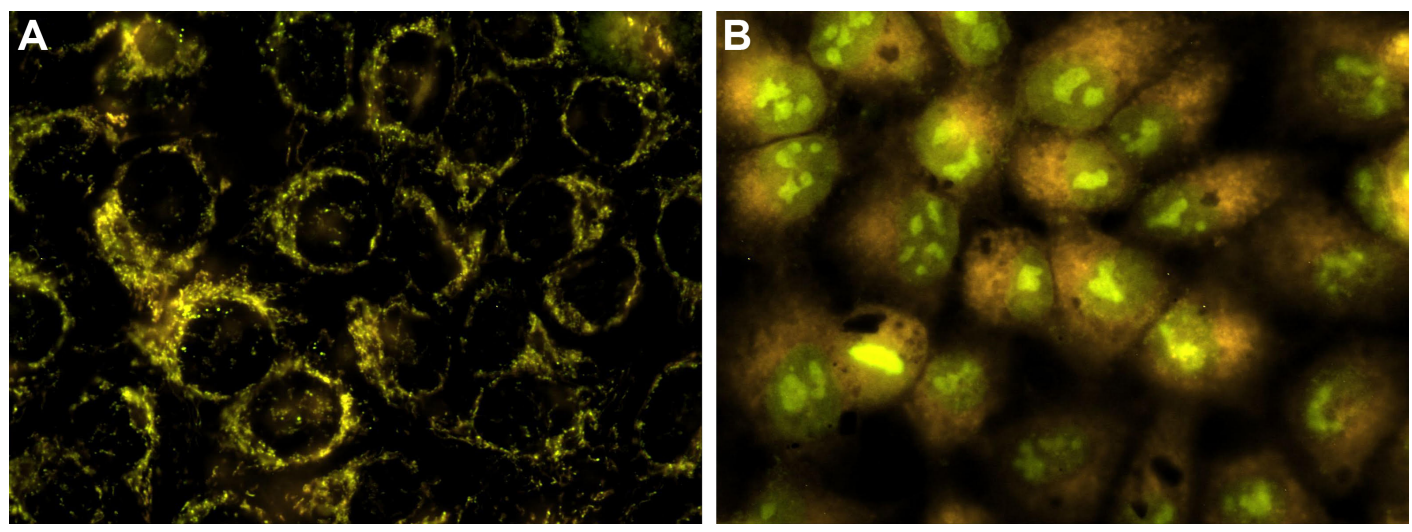

Figure 9 ROS generation in HeLa cells after $24 \mathrm{~h}$ exposure to saline (A) or the nanoparticles sample (B). Abbreviation: ROS, reactive oxygen species.

of the iron oxide nanoparticles, the better. In this sense, the synthesized SPIONs met the basic requirements for the nanoparticles for theranostic applications. Further tests were required to check if the sample had featured properties that could enhance its therapeutic potential by providing heavy impact on the tumor when combined with additional targeted manipulations (ultrasound, magnetic field, and so on).

It is assumed that the property of the formulation as granulation may fortify its therapeutic effects, because these nanoparticles appear to be capable of attacking cancer cells directly in their nuclei. This represents a great additive effect for thermal therapy and other SPION-based therapeutic approaches. It should be noted that this nuclear ROS generation might arise from a chemical factor other than the iron oxide core itself. This, however, does not compromise the formulation but rather sets another question concerning the mechanisms of action of this novel nanosystem. These mechanisms are a subject for future studies.

\section{Conclusion}

Low toxic superparamagnetic $\mathrm{Fe}^{3+}$ oxide nanoparticles of nearly spherical in shape with maghemite structure for theranostic applications in oncology were synthesized by fast enough microwave-assisted coprecipitation and fully characterized by TEM, DLS, XRD, XPS, XANES, magnetometry, and Mossbauer spectroscopy. The SPIONs under study did not have significant effects on the viability of the HeLa cells, however, caused a profound increase in ROS generation in the HeLa cells - both in the cytoplasm and the nucleus. Thus, in addition to the standard theranostic characteristics, the present $\gamma$ - $\mathrm{Fe}_{2} \mathrm{O}_{3}$ nanoparticles could be used for multimodal (both local magnetic hyperthermia and generation of ROS) anticancer therapy.

\section{Acknowledgments}

This work was financially supported by the Ministry of Education and Science of the Russian Federation (agreement no 14.587.21.0027, unique identifier RFMEFI58716×0027). The XAS experiments were performed at beamline ID26 of ESRF, Grenoble, France. We are grateful to Sara Lafuerza and Pieter Glatzel at the ESRF for providing assistance in using beamline ID26.

\section{Disclosure}

The authors report no conflicts of interest in this work.

\section{References}

1. Golovin YI, Gribanovsky SL, Golovin DY, et al. Towards nanomedicines of the future: remote magneto-mechanical actuation of nanomedicines by alternating magnetic fields. J Control Release. 2015; 219:43-60.

2. Ali A, Zafar H, Zia M, et al. Synthesis, characterization, applications, and challenges of iron oxide nanoparticles. Nanotechnol Sci Appl. 2016; 19(9):49-67.

3. Nguyen DT, Kim K-S. Controlled magnetic properties of iron oxidebased nanoparticles for smart therapy. Kona Powder Part J. 2016;33: 33-47.

4. Revia RA, Zhang M. Magnetite nanoparticles for cancer diagnosis, treatment, and treatment monitoring: recent advances. Mater Today. 2016;19(3):157-168.

5. Kandasamy G, Maity D. Recent advances in superparamagnetic iron oxide nanoparticles (SPIONs) for in vitro and in vivo cancer nanotheranostics. Int J Pharm. 2015;496(2):191-218.

6. Singh A, Sahoo SK. Magnetic nanoparticles: a novel platform for cancer theranostics. Drug Discov Today. 2014;19(4):474-481.

7. Shubayev VI, Pisanic TR 2nd, Jin S. Magnetic nanoparticles for theranostics. Adv Drug Deliv Rev. 2009;61(6):467-477.

8. Zanganeh S, Hutter G, Spitler R, et al. Iron oxide nanoparticles inhibit tumour growth by inducing pro-inflammatory macrophage polarization in tumour tissues. Nat Nanotechnol. 2016;11(11):986-994.

9. Iacovita C, Florea A, Dudric R, et al. Small versus large iron oxide magnetic nanoparticles: hyperthermia and cell uptake properties. Molecules. 2016;21(10):E1357.

10. Zarschler K, Rocks L, Licciardello N, et al. Ultrasmall inorganic nanoparticles: state-of-the-art and perspectives for biomedical applications. Nanomedicine. 2016;12(6):1663-1701. 
11. Lee JH, Ju JE, Kim BI, et al. Rod-shaped iron oxide nanoparticles are more toxic than sphere-shaped nanoparticles to murine macrophage cells. Environ Toxicol Chem. 2014;33(12):2759-2766.

12. Singh N. Conference scene - nanotoxicology: health and environmental impacts. Nanomedicine (Lond). 2009;4(4):385-390.

13. Li L, Jiang L-L, Zeng Y, Liu G. Toxicity of superparamagnetic iron oxide nanoparticles: research strategies and implications for nanomedicine. Chin Phys B. 2013;22(12):127503.

14. Gaharwar U, Paulraj R. Iron oxide nanoparticles induced oxidative damage in peripheral blood cells of rat. J Biomed Sci Eng. 2015;8:274-286.

15. Laurent S, Forge D, Port M, et al. Magnetic iron oxide nanoparticles: synthesis, stabilization, vectorization, physicochemical characterizations, and biological applications. Chem Rev. 2008;108(6):2064-2110.

16. Blanco-Andujar C, Ortega D, Southern P, Pankhurst QA, Thanh NTK. High performance multi-core iron oxide nanoparticles for magnetic hyperthermia: microwave synthesis, and the role of core-to-core interactions. Nanoscale. 2015;7(5):1768-1775.

17. Glatzel P, Bergmann U. High resolution 1s core hole X-ray spectroscopy in $3 \mathrm{~d}$ transition metal complexes - electronic and structural information. Coord Chem Rev. 2005;249:65-95.

18. Glatzel P, Weng T-C, Kvashnina K, et al. Reflections on hard X-ray photon-in/photon-out spectroscopy for electronic structure studies. J Electron Spectros Relat Phenomena. 2013;188:17-25.

19. Chen LX, Liu T, Thurnauer MC, Csencsits R, Raj T. Fe $\mathrm{O}_{3}$ nanoparticle structures investigated by X-ray absorption near-edge structure, surface modifications, and model calculations. J Phys Chem B. 2002;106(34): 8539-8546.
20. Piquer C, Laguna-Marco MA, Roca AG, Boada R, Guglieri C, Chaboy J. Fe K-edge X-ray absorption spectroscopy study of nanosized nominal magnetite. J Phys Chem C. 2014;118:1332-1346.

21. Matsnev ME, Rusakov VS. SpectrRelax: an application for Mössbauer spectra modeling and fitting. AIP Conf Proc. 2012;1489:178-185.

22. Kozakov AT, Kochur AG, Googlev KA, et al. X-ray photoelectron study of the valence state of iron in iron - containing single - crystal $\left(\mathrm{BiFeO}_{3}, \mathrm{PbFe}_{1 / 2} \mathrm{Nb}_{1 / 2} \mathrm{O}_{3}\right)$, and ceramic $(\mathrm{BaFe} 1 / 2 \mathrm{Nb} 1 / 2 \mathrm{O} 3)$ multiferroics. J Electron Spectros Relat Phenomena. 2011;184:16-23.

23. Yamashita T, Hayes P. Analysis of XPS spectra of Fe $2+$ and Fe3+ ions in oxide materials. Appl Surf Sci. 2008;254:2441-2449.

24. Chuev MA. On the shape of GammaResonance Spectra of ferrimagnetic nanoparticles under conditions of metamagnetism. JETP Lett 2013;98(8):465-470.

25. Menil F. Systematic trends of the ${ }^{57} \mathrm{Fe}$ Mossbauer isomer shifts in $\left(\mathrm{FeO}_{\mathrm{n}}\right)$ and $\left(\mathrm{FeF}_{\mathrm{n}}\right)$ polyhedra. Evidence of a new correlation between the isomer shift and the inductive effect of the competing bond T-X $\left({ }^{*} \mathrm{Fe}\right)$ (where $\mathrm{X}$ is $\mathrm{O}$ or $\mathrm{F}$ And $\mathrm{T}$ any element with a formal positive charge). J Phys Chem Solids. 1985;46(7):763-789.

26. Kwon IK, Lee SC, Han B, Park K. Analysis on the current status of targeted drug delivery to tumors. J Control Release. 2012;164(2): $108-114$.

27. Zhang J, Lou X, Jin L, et al. Necrosis, and then stress induced necrosislike cell death, but not apoptosis, should be the preferred cell death mode for chemotherapy: clearance of a few misconceptions. Oncoscience. 2014;1(6):407-422.
International Journal of Nanomedicine

\section{Publish your work in this journal}

The International Journal of Nanomedicine is an international, peerreviewed journal focusing on the application of nanotechnology in diagnostics, therapeutics, and drug delivery systems throughout the biomedical field. This journal is indexed on PubMed Central, MedLine, CAS, SciSearch $\AA$, Current Contents $₫ /$ Clinical Medicine,

\section{Dovepress}

Journal Citation Reports/Science Edition, EMBase, Scopus and the Elsevier Bibliographic databases. The manuscript management system is completely online and includes a very quick and fair peer-review system, which is all easy to use. Visit http://www.dovepress.com/ testimonials.php to read real quotes from published authors. 